\title{
Non-linear inelastic analysis of steel arches at elevated temperatures
}

\author{
Amin Heidarpour*, Abdul Azim Abdullah, Mark A. Bradford \\ Centre for Infrastructure Engineering and Safety, School of Civil and Environmental Engineering, The University of New South Wales, UNSW Sydney, NSW 2052, Australia
}

\section{A R T I C L E I N F O}

\section{Article history:}

Received 5 August 2009

Accepted 9 October 2009

\section{Keywords:}

Steel arch

Non-discretisation model

Non-linear material

Geometric non-linearity

Elevated temperatures

\begin{abstract}
A B S T R A C T
An innovative non-discretisation mechanical-based method is developed in this paper to analyse a steel arch at elevated temperatures so that its behaviour can be quantified. The steel arch has a generic but singly-symmetric cross-section with elastic and plastic parts, and it is subjected to an arbitrary thermal profile which varies along the length of the arch as well as through the depth of the cross-section. The effects of geometric and material non-linearity as well as potential catenary action which can occur at high temperatures are taken into account in the formulation. The efficiency and accuracy of the generic model developed is demonstrated by a comparison with a finite element model undertaken using ABAQUS. The proposed method is then utilised to elucidate some significant factors, such as the magnitude of the temperature at bottom fibre of the cross-section as well as the ratio of the temperature at the top fibre to that at the bottom fibre, on the response of a steel arch member during fire loading. The proposed model provides a computationally superior formulation to that of commercial finite element packages, and forms a platform which can be used for structural steel arch design and evaluation in the development of codified approaches to fire design on a performance basis.
\end{abstract}

(C) 2009 Elsevier Ltd. All rights reserved.

\section{Introduction}

Steel arches find widespread deployment in engineering practice, in applications such as large span roofing structures which can compromise life, limb and amenity in the event of a fire. Unlike research on steel rectangular-framed structures that are subjected to elevated temperatures which has been quite plentiful in recent years, comprehensive investigations of the non-linear behaviour of steel arches under fire loading have not been reported. Since an arch is often connected with other structural members such as roof sheeting, lateral bracing, and purlins that produce sufficient restraining actions to the arch to prevent out-of-plane buckling [1], it is essential to develop a significant understanding of the in-plane inelastic behaviour of steel arch members at elevated temperatures so that a safe and economic designs can be achieved for the fire limit state.

Geometrical and material non-linearities are the two significant factors that affect the behaviour of structures at elevated temperatures. The effect of geometrical non-linearity arises when the deformations are large enough to cause significant changes in the geometry of the structure, so that the equilibrium equations must be formulated with respect to the deformed shape of the structure $[2,3]$ (of course, taking the geometrical non-linearity into account and the use of second-order analysis are always necessary for

\footnotetext{
* Corresponding author. Tel.: +612 9385 5029; fax: +61293859747.

E-mail address: a.heidarpour@unsw.edu.au (A. Heidarpour).
}

the stability consideration of structures). On the other hand, material non-linearity arises when yielding occurs or the stress-strain behaviour exhibits non-linear characteristics which result in yielding spreading through the cross-section and along the length of the member [4]. Basically, there are two approaches for including the effect of material non-linearity into non-linear structural analysis: viz. the plastic hinge approach and the plastic zone approach. Recognising that accurate determination of the thermal regime is difficult and often not justified, the adequacy of approximate solutions based on the plastic hinge concept model has been argued, for which inelasticity is developed in zero-length hinges at the member ends with the remainder of the member being elastic [4,5]. This formulation can lead to efficient and robust solutions, but they are known to be approximate. On the other hand, plastic zone models [6] which allow for gradual yield propagation through the crosssection and along the length are accurate, but possess too many structural freedoms and Gauss-Legendre integration points to render them applicable to large frames, and so adaptive techniques such as those described previously $[7,8]$ have evolved.

Structures can be considered as being subjected to a constant (or sustained) level of load when exposed to fire, but with a degradation of the material properties with increasing temperature. Unlike at the ambient state for which extensive studies have been reported using analytical or numerical methods to analyse the inplane buckling of steel arches, the non-linear behaviour of steel arches under thermal loading has hitherto been little investigated. Wood and Zienkiewicz [9] investigated the non-linear behaviour of elastic in-plane oriented bodies such as beams, frames and 